\title{
IMPLICATIVE SEMI-LATTICES
}

\author{
BY \\ WILLIAM C. NEMITZ( $\left.{ }^{1}\right)$
}

1. Introduction. An implicative semi-lattice is an algebraic system havingas models logical systems equipped with implication and conjunction, but not possessing a disjunction. The position of implicative semi-lattices in algebraic logic is clearly displayed in [2]. In [1], the relation of implicative lattices to Brouwerian logics is explained. In the terminology of [1], an implicative lattice would be called a relatively pseudo-complemented lattice. Monteiro [6] and Glivenko [5] have each given a set of axioms for Brouwerian logic. Glivenko has also proved certain results for Brouwerian logics (equipped with disjunction) which are proved for implicative semi-lattices in $\$ 4$ of this paper. In [3] and [7], the relation of implicative lattices to closure algebras and topologies is explored.

There are four principal results in this paper. The first (Theorem 3.2) states that the relationship between homomorphisms of implicative semi-lattices and their kernels is that usually found in algebra, and not found in lattice theory, namely, that every homomorphism is essentially determined by its kernel. In [4], Frink has shown that the structure of a pseudo-complemented semi-lattice, and therefore of a bounded implicative semi-lattice, is very similar to that of a bounded implicative lattice, in that the semi-lattice of "closed" elements is a Boolean algebra, despite the absence of a least upper bound. The second result of this paper (Theorem 4.4) states that every element of a bounded implicative semi-lattice is the meet of its closure with a dense element, a result which is well known for bounded implicative lattices. The third result (Theorem 5.2) gives a method for constructing all bounded implicative semi-lattices having a given Boolean algebra for closed algebra, and a given implicative semi-lattice for dense filter; the fourth result (Theorem 6.6) gives essentially the same information about homomorphisms.

2. Implicative semi-lattices. An implicative semi-lattice is a system $\langle L, \leqq, \wedge, *\rangle$ in which $L$ is a nonempty set, $\leqq$ is a partial order on $L, \wedge$ is a greatest lower bound with respect to $\leqq$, and $*$ is a binary composition in $L$ such that for any elements $x, y$, and $z$ of $L$,

Received by the editors October 8, 1963.

( $\left.{ }^{1}\right)$ The research reported in this paper was done while the author was the recipient of a National Science Foundation research participation award at the University of Oklahoma during the summer of 1963. 
$z \leqq x * y$ if and only if $z \wedge x \leqq y$.

The operation $*$ is called implication. We shall usually refer to $L$ as an implicative semi-lattice omitting reference to the order and compositions.

LeMmA 2.1. Let $L$ be an implicative semi-lattice. Then L has a greatest element, denoted by 1 . Also the following results are true for any elements $x, y$, and $z$ of $L$ :

(i) $x \leqq 1, x * x=1, x=1 * x$;

(ii) $x \leqq y$ if and only if $x * y=1$;

(iii) $y \leqq x * y$;

(iv) if $x \leqq y$, then $x * z \geqq y * z$, and $z * x \leqq z * y$;

(v) $x *(y * z)=(x \wedge y) * z$;

(vi) $x *(y \wedge z)=x * y \wedge x * z$

(vii) $x *(y * z)=(x * y) *(x * z)$;

(viii) if $L$ is a lattice with least upper bound $V$, then $L$ is distributive, and $(x \vee y) * z=(x * z) \wedge(y * z)$.

Proof. All of these results except (vii) are proved in [2]. Also the inequality

$$
x *(y * z) \leqq(x * y) *(x * z)
$$

is proved in the same place. To prove the other half of (vii), let $u \in L$. If $u \leqq(x * y) *(x * z)$, then $u \wedge(x * y) \leqq x * z$, so $u \wedge x \wedge(x * y) \leqq z$. But by (iii), $u \wedge x \wedge y \leqq u \wedge x \wedge(x * y)$, so by $(v), u \leqq(x \wedge y) * z=x *(y * z)$.

3. Homomorphisms. A nonempty subset $J$ of an implicative semi-lattice $L$ is a filter of $L$ and only if

(i) $x \wedge y \in J$ for any $x \in J$ and $y \in J$, and

(ii) if $x \in J$ and $y \in L$, and $y \geqq x$, then $y \in J$.

A maximal proper filter is called an ultrafilter.

LEMMA 3.1. Let $L$ and $L^{\prime}$ be two implicative semi-lattices. Let $\alpha$ be a function from Lonto $L^{\prime}$, such that

$$
\alpha(x * y)=\alpha(x) * \alpha(y),
$$

for all $x$ and $y$ elements of L. Let $J=\alpha^{-1}(1)$. Then

(i) $\alpha(1)=1$;

(ii) $\alpha$ is isotone;

(iii) $\alpha(x \wedge y)=\alpha(x) \wedge \alpha(y)$ for any elements $x$ and $y$ of $L$ if and only if $J$ is a filter;

(iv) $\alpha$ is an isomorphism if and only if $J=\{1\}$;

(v) if $L$ and $L^{\prime}$ are both lattices, and if $J$ is a filter, then denoting least upper bounds by $\vee, \alpha(x \vee y)=\alpha(x) \vee \alpha(y)$, for any elements $x$ and $y$ of $L$. 
Proof. (i) $\alpha(1)=\alpha(1 * 1)=\alpha(1) * \alpha(1)=1$.

(ii) If $x \leqq y$, then $\alpha(x) * \alpha(y)=\alpha(x * y)=\alpha(1)=1$, so $\alpha(x) \leqq \alpha(y)$.

(iii) Clearly, if $\alpha(x \wedge y)=\alpha(x) \wedge \alpha(y)$ for any elements $x$ and $y$ of $L$, then $J$ is a filter. Assume therefore that $J$ is a filter, and let $x$ and $y$ be any elements of $L$. By (ii), $\alpha(x \wedge y) \leqq \alpha(x) \wedge \alpha(y)$. Now assume that $z$ is any element of $L^{\prime}$ such that $z \leqq \alpha(x)$, and $z \leqq \alpha(y)$. Since $\alpha$ is onto, there is some $u \in L$ such that $z=\alpha(u)$. So $u *(x \wedge y)=(u * x) \wedge(u * y) \in J$, and thus $z=\alpha(u) \leqq \alpha(x \wedge y)$. So $\alpha(x \wedge y)$ $=\alpha(x) \wedge \alpha(y)$.

(iv) It suffices to show that if $J=\{1\}$, then $\alpha(x) \leqq \alpha(y)$ implies $x \leqq y$ for any elements $x$ and $y$ of $L$. But if $\alpha(x) \leqq \alpha(y)$, then $x * y$ is an element of $J$, and so $x * y=1$, which implies that $x \leqq y$.

(v) Clearly $\alpha(x) \vee \alpha(y) \leqq \alpha(x \vee y)$ for any elements $x$ and $y$ of $L$. Let $z \in L^{\prime}$ such that $z \geqq \alpha(x)$ and $z \geqq \alpha(y)$. Let $u$ be an element of $L$ such that $\alpha(u)=z$. Then

$$
(x \vee y) * u=(x * u) \wedge(y * u) \in J,
$$

and so $\alpha(x \vee y) \leqq z$. Thus $\alpha(x \vee y)=\alpha(x) \vee \alpha(y)$.

If $\alpha$ is a function from an implicative semi-lattice onto an implicative semilattice as described in Lemma 3.1, then by the kernel of $\alpha$, we shall mean the set $\alpha^{-1}(1)$. Hereafter we shall be concerned only with functions from implicative semi-lattices on to implicative semi-lattices which satisfy the conditions of Lemma 3.1, and have filters for kernels. We shall refer to such functions as homomorphisms, and if any additional structure is present and preserved, a suitable modifier will be added (for example, lattice homomorphism, Boolean (algebra) homomorphism, etc.).

Let $J$ be a filter of an implicative semi-lattice $L$. We may define an equivalence relation on $L$ by saying that $x$ is equivalent to $y$ if and only if there is some element $c$ of $J$ such that

$$
x \wedge c=y \wedge c .
$$

For $x \in L$, let $x / J$ denote the equivalence class of $x$, and let $L / J$ denote the quotient structure defined by this equivalence relation. We may make $L / J$ into a semilattice by setting

$$
\begin{aligned}
& \therefore \because, \ldots, \therefore, \ldots=\because * x
\end{aligned}
$$

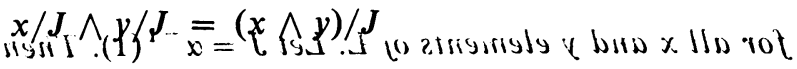

and saying $x / J \leqq y / J$ if and only if

$$
i I=(1) \times \quad \text { (i) }
$$

$$
x / J \wedge y / J=x / J J_{0}(v) \wedge \wedge(x) x=(v \wedge x) x \text { (iii) }
$$

THEOREM 3.2. Let $L$ be an implicative semi-lattice, and let $J$ besteffidter

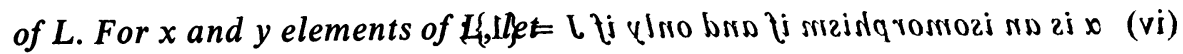

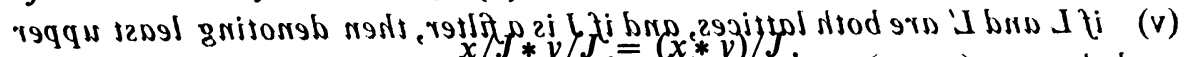

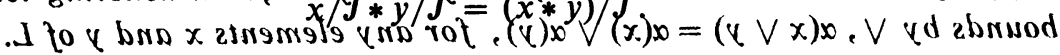


Then $L / J$ is an implicative semi-lattice with * as implication, and the mapping from Lonto $L / J$ that assigns to each element of $L$ its equivalence class in $L / J$ is a homomorphism. Thus, $J$ is a filter of $L$ if and only if $J$ is the kernel of a homomorphism. Furthermore, if $\alpha$ is a homomorphism from $L$ onto $L^{\prime}$ with kernel $J$, then $L^{\prime}$ is isomorphic to $L / J$.

Proof. We must show that $*$ is well defined on $L / J$, that it is an implication on $L / J$, and that if $\alpha$ is any homomorphism on $L$ with kernel $J$, and if $x$ and $y$ are any elements of $L$, then $\alpha(x)=\alpha(y)$ if and only if there is some $c \in J$, such that $x \wedge c=y \wedge c$. To show that $*$ is well defined, let $x, y, u$, and $v$ be elements of $L$ such that there are elements $c_{1}$ and $c_{2}$ of $J$ such that $x \wedge c_{1}=u \wedge c_{1}$ and $y \wedge c_{2}=v \wedge c_{2}$. We want to find some $c \in J$ such that

$$
(x * y) \wedge c=(u * v) \wedge c .
$$

Clearly

$$
c_{1} \wedge\left(\left(x \wedge c_{1}\right) *\left(y \wedge c_{2}\right)\right)=c_{1} \wedge\left(\left(u \wedge c_{1}\right) *\left(v \wedge c_{2}\right)\right)
$$

But

$$
c_{1} \wedge\left(\left(x \wedge c_{1}\right) *\left(y \wedge c_{2}\right)\right)=c_{1} \wedge\left(\left(c_{1} *(x * y)\right) \wedge\left(x *\left(c_{1} * c_{2}\right)\right)\right)
$$

Now clearly

$$
c_{1} \wedge\left(c_{1} *(x * y)\right) \leqq c_{1} \wedge(x * y)
$$

But since

$$
(x * y) \leqq c_{1} *(x * y),
$$

we have that

$$
c_{1} \wedge(x * y) \leqq c_{1} \wedge\left(c_{1} *(x * y)\right)
$$

So

$$
c_{1} \wedge\left(c_{1} *(x * y)\right)=c_{1} \wedge(x * y) \text {. }
$$

Also since $c_{2} \in J, J$ is a filter, and

$$
c_{2} \leqq c_{1} * c_{2} \leqq x *\left(c_{1} * c_{2}\right),
$$

so $x *\left(c_{1} * c_{2}\right) \in J$. Let

$$
\bar{c}_{1}=c_{1} \wedge\left(x *\left(c_{1} * c_{2}\right)\right)
$$

$\operatorname{Phen}^{1} \bar{c}_{1} \in \boldsymbol{J}$, and

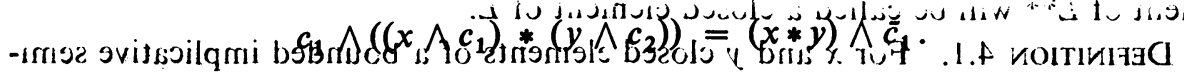

Similarly there is an element $\bar{c}_{2}$ of $J$ such that

fol eכiftsil

$$
\left.c_{1} \wedge\left(\left(u \wedge \dot{c}_{1}^{*}\right)^{*} *\left(v \wedge^{*} x\right)=\stackrel{\psi}{ } \wedge_{2}\right)\right)(\stackrel{x}{=} * v) \wedge \bar{c}_{2} \text {. }
$$

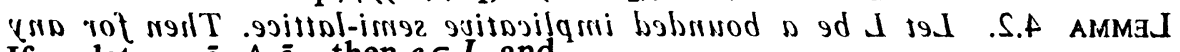
If we let $c=\bar{c}_{1} \wedge \bar{c}_{2}$, then $c \in J$, and

a to $<$ bono $x$ asirgmals

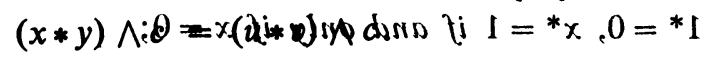


To show that $*$ is an implication on $L / J$, first note that for any elements $x$ and $y$ of $L, x / J \leqq y / J$ if and only if $x / J \wedge y / J=x / J$ which is true if and only if there is some element $c$ of $J$ such that $x \wedge c \leqq y$. Now let $u$ be an element of $L$. Then $u / J \leqq(x * y) / J$ if and only if there is some $c \in J$ such that $u \wedge c \leqq x * y$, which is true if and only if $u \wedge x \wedge c \leqq y$, which is true if and only if $u / J \wedge x / J \leqq y / J$. So $*$ is an implication on $L / J$.

Now let $\alpha$ be any homomorphism with domain $L$, and kernel $J$. For any elements $x$ and $y$ of $L, \alpha(x)=\alpha(y)$ if and only if

$$
(x * y) \wedge(y * x) \in J .
$$

Assume that $\alpha(x)=\alpha(y)$. We want an element $c$ of $J$ such that

Now

$$
x \wedge c=y \wedge c .
$$

$$
x \wedge(x * y) \wedge(y * x) \leqq y \wedge(y * x)
$$

so

Similarly

$$
x \wedge(x * y) \wedge(y * x) \leqq y \wedge(y * x) \wedge(x * y)
$$

so

$$
y \wedge(y * x) \wedge(x * y) \leqq x \wedge(x * y) \wedge(y * x),
$$

$$
x \wedge(x * y) \wedge(y * x)=y \wedge(x * y) \wedge(y * x),
$$

and thus $(x * y) \wedge(y * x)$ is the desired element of $J$. Now assume that there is some element $c$ of $J$ such that $x \wedge c=y \wedge c$. Then $x \wedge c \leqq y$, so $c \leqq x * y$. Similarly $c \leqq y * x$, so

$$
c \leqq(x * y) \wedge(y * x),
$$

and thus $(x * y) \wedge(y * x) \in J$. Hence $\alpha(x)=\alpha(y)$.

4. Bounded implicative semi-lattices. An implicative semi-lattice $L$ is bounded if and only if it has an element denoted by 0 , such that $0 \neq 1$, and $x \geqq 0$ for every $x$ an element of $L$. In this case, for $x \in L$, let $x^{*}=x * 0$. Then $x^{*}$ plays the role of a pseudo-complement of $x$ in the sense of [4]. For any bounded implicative semi-lattice $L$, and any subset $X$ of $L, X^{* *}$ will denote that set of elements $x$ of $L$ such that there is some element $y$ of $L$ satisfying $x=y^{* *}$. An element of $L^{* *}$ will be called a closed element of $L$.

Definition 4.1. For $x$ and $y$ closed elements of a bounded implicative semilattice, let

$$
x \vee y=\left(x^{*} \wedge y^{*}\right)^{*}
$$

LEMMA 4.2. Let $L$ be a bounded implicative semi-lattice. Then for any elements $x$ and $y$ of $L$,

(i) $1^{*}=0, x^{*}=1$ if and only if $x=0$; 
(ii) if $x \leqq y$, then $x^{*} \geqq y^{*}$ and $x^{* *} \leqq y^{* *}$;

(iii) $x \leqq x^{* *}, x^{*}=x^{* * *}$, if $x$ is closed, then $x=x^{* *}$;

(iv) $(x \wedge y)^{* *}=x^{* *} \wedge y^{* *}$;

(v) $(x \wedge y)^{*}=x * y^{*}$;

(vi) if $y$ is closed, then so is $x * y$, and in fact $x * y=x^{*} \vee y=x^{* *} * y$.

Proof. (i) is obvious. (ii), (iii) and (iv) are proved in [4]. To prove (v), note that for any $u \in L, u \leqq(x \wedge y)^{*}$ if and only if $u \wedge x \wedge y=0$, which is equivalent to $u \wedge x \leqq y^{*}$. So $u \leqq(x \wedge y)^{*}$ if and only if $u \leqq x * y^{*}$. To prove (vi), it suffices to prove that $x * y=x^{*} \vee y$. But by (iii), (iv) and (v), we have that

$$
x^{*} \vee y=\left(x^{* *} \wedge y^{*}\right)^{*}=\left(x \wedge y^{*}\right)^{*}=x * y^{* *}=x * y .
$$

In [4], Frink has proved the following theorem in the more general context of a pseudo-complemented semi-lattice.

TheOREM 4.3 (GlivenKo-Frink). Let L be a bounded implicative semi-lattice. Then $L^{* *}$ is a Boolean algebra with $\bigvee$ as least upper bound and *as complement.

We shall use (**) to denote the mapping from $L$ onto $L^{* *}$ given by

$$
(* *)(x)=x^{* *},
$$

for all $x \in L$. Let $D$ be the kernel of this mapping. Then $d \in D$ if and only if $d^{* *}=1$, which is true if and only if $d^{*}=0$. From Lemma 4.2(ii) and (iv), it follows that $D$ is a filter of $L$. Also, for $d \in D$ and $x \in L$, since $d \leqq x * d, x * d \in D$. Therefore $D$ is an implicative semi-lattice. We shall call $D$ the dense filter of $L$, and shall say that an element $d$ of $L$ is dense if and only if $d \in D$.

THEOREM 4.4. Let L be a bounded implicative semi-lattice. For any element $x$ of $L$, there is a dense element $d$ such that

$$
x=x^{* *} \wedge d
$$

In fact $x^{* *} * x$ is the greatest such dense element.

Proof. Since $x \leqq x^{* *}$, and $x \leqq x^{* *} * x, x \leqq x^{* *} \wedge\left(x^{* * *} * x\right)$. But $x^{* *} \wedge\left(x^{* *} * x\right)$ $\leqq x$, so

$$
x=x^{* *} \wedge\left(x^{* * * x)}\right. \text {. }
$$

Also if $x=x^{* *} \wedge d$, then $d \leqq x^{* *} * x$. It remains to show that $x^{* * * x}$ is dense. From (1) and Lemma 4.2 (iv), we get that

$$
x^{* *}=x^{* *} \wedge\left(x^{* * * x}\right)^{* *},
$$

and so $\left(x^{* * * x}\right)^{*} \leqq x^{*}$. But $x^{*} \wedge x^{* *} \leqq x$, so $x^{*} \leqq x^{* * * x}$, and hence $\left(x^{* *} * x\right)^{*} \leqq x^{* *}$. Therefore 


$$
\left(x^{* *} * x\right)^{*} \leqq x^{*} \wedge x^{* *}=0,
$$

and so $x^{* *} * x$ is dense.

CoRollary 4.5. Let $x$ and $y$ be elements of a bounded implicative semilattice L, such that $x^{* *}=y^{* *}$. Then there is a dense element $d$ such that $x \wedge d=y \wedge d$.

TheOREM 4.6. Let L be a bounded implicative semi-lattice. Then the mapping (**) is a homomorphism from Lonto $L^{* *}$.

Proof. The only thing which needs proof is that $(x * y)^{* *}=x^{* *} * y^{* *}$ for any elements $x$ and $y$ of $L$. But by Theorem 4.4, there is a dense element $d$ such that $y=y^{* *} \wedge d$. Hence by Lemma 4.2 , since $x * d$ is dense,

$$
(x * y)^{* *}=\left(x *\left(y^{* *} \wedge d\right)\right)^{* *}=\left(\left(x * y^{* *}\right) \wedge(x * d)\right)^{* *}=x * y^{* *}=x^{* * *} y^{* *} .
$$

COROLlaRy 4.7. Let $x$ be an element of a bounded implicative semi-lattice L. Then $x$ is dense if and only if there is some element $y$ of L such that $x=y^{* *} * y$.

The simple Boolean algebra plays a central role in the algebraic theory of classical logic. We now characterize all bounded implicative semi-lattices, the closed algebras of which are simple.

Definition 4.8. Let $L$ be a bounded implicative semi-lattice. We say that $L$ is artificially bounded if and only if $L-\{0\}$ is an implicative semi-lattice.

THEOREM 4.9. Let $L$ be a bounded implicative semi-lattice. Then the closed algebra of $L$ is simple if and only if $L$ is artificially bounded. That $L$ is artificially bounded is also equivalent to the assertion that every nonzero element of $L$ is dense.

Proof. The equivalence of the simplicity of $L^{* *}$ with the assertion that every nonzero element of $L$ is dense follows from Theorem 4.4. Furthermore, since the dense filter of $L$ is an implicative semi-lattice, that every nonzero element of $L$ is dense implies that $L$ is artificially bounded. Now assume that $L$ is artificially bounded, and let $x$ be a nonzero element of $L$. Then for $y$ any element of $L, x \wedge y=0$ if and only if $y=0$. So $x^{*}=0$, and hence $x$ is dense.

5. Construction of bounded implicative semi-lattices. In this section we shall be concerned with a Boolean algebra $\left\langle A, \leqq, \wedge, \vee, 0,1,{ }^{\prime}\right\rangle$ and an implicative semilattice $\langle D, \leqq, \wedge, *\rangle$. We shall use the same notations for the orders and operations in $A$ and $D$, since context will indicate clearly which system involved. We wish to characterize all bounded implicative semi-lattices having $A$ for closed algebra and $D$ for dense filter. We shall be concerned with certain functions from $A \times D$ into $D$. For such a function $f$ and for each element $a$ of $A$, there is defined a function $f_{a}$ from $D$ into $D$ such that 


$$
f_{a}(d)=f(a, d)
$$

for each $d \in D$. Each such function $f$ and element $a \in A$ determines an equivalence relation on $D$. That is for $d$ and $e$ elements of $D, d$ is $a$-equivalent to $e$ if and only if

$$
f_{a}(d)=f_{a}(e) .
$$

We shall call the equivalence classes of this relation $a$-classes of $f$.

Definition 5.1. If $A$ is a Boolean algebra and $D$ is an implicative semi-lattice, a function $f$ from $A \times D$ into $D$ is admissible if and only if for every element $a$ of $A, f_{a}$ is an endomorphism on $D$, and the following three conditions are satisfied, for all elements $a$ and $b$ of $A$, and any element $d$ of $D$ :

(i) $f(a \wedge b, d)=f(a, f(b, d))$;

(ii) if $a \leqq b$, then $f(a, d) \geqq f(b, d)$;

(iii) $f(0, d)=1, f(1, d)=d$.

THEOREM 5.2. Let $A$ be a Boolean algebra, and let $D$ be an implicative semi-lattice. Let $f$ be an admissible function from $A \times D$ into $D$. Let $\theta$ be the equivalence relation on $A \times D$ defined $b y\langle a, d\rangle \theta\langle b, e\rangle$ if and only if

and

$$
a=b
$$

$$
f(a, d)=f(a, e)
$$

for $a$ and $b$ elements of $A$, and $d$ and e elements of $D$. Let $[a, d]$ denote the equivalence class of $\langle a, d\rangle$, and for $[a, d]$ and $[b, e]$ elements of $(A \times D) / \theta$, let

$$
[a, d] \wedge[b, e]=[a \wedge b, d \wedge e]
$$

and

We say

$$
[a, d] *[b, e]=\left[a^{\prime} \vee b, f(a, d * e)\right]
$$

$$
[a, d] \leqq[b, e]
$$

if and only if

$$
[a, d] \wedge[b, e]=[a, d] .
$$

Then with these operations, $(A \times D) / \theta$ is a bounded implicative semi-lattice, the closed algebra of which is isomorphic to $A$, and the dense filter of which is isomorphic to $D$. Also for any $a \in A$ and $d \in D, f(a, d)$ corresponds to thidi * composition of the image of $a$ in $(A \times D) / \theta$ with that of $d$. Furthermore, if $L$ is a bounded implicative semi-lattice with dense filter $D$, and if $f$ is the function. from $L^{* *} \times D$ into $D$ defined by 


$$
f(a, d)=a * d
$$

for $a \in A$ and $d \in D$, then $f$ is admissible and $L$ is isomorphic to $\left(L^{* *} \times D\right) / \theta$.

Proof. Clearly $\theta$ is an equivalence relation on $A \times D$. We now establish two simple facts which will be useful in the rest of the proof.

(i) Assume that $a$ and $b$ are elements of $A, a \geqq b$, and that $d$ and $e$ are elements of $D$ such that $f(a, d)=f(a, e)$. Then

$f(b, d)=f(a \wedge b, d)=f(b, f(a, d))=f(b, f(a, e))=f(a \wedge b, e)=f(b, e)$.

So $f(b, d)=f(b, e)$.

(ii) For any elements $a$ and $b$ of $A$ and elements of $d$ and $e$ of $D,[a, d] \leqq[b, e]$ if and only if

$$
[a, d] \wedge[b, e]=[a, d],
$$

which is true if and only if $a \leqq b$ and $f(a, d) \leqq f(a, e)$.

We now show that $\wedge$ is well defined. Suppose that $a, b$, and $c$ are elements of $A$, and that $d_{1}, d_{2}$, and $d_{3}$ are elements of $D$ and that $\left[a, d_{1}\right]=\left[c, d_{3}\right]$. Then $a=c$ and $f\left(a, d_{1}\right)=f\left(a, d_{3}\right)$. Hence by (i),

$$
\begin{aligned}
f\left(a \wedge b, d_{1} \wedge d_{2}\right) & =f\left(a \wedge b, d_{1}\right) \wedge f\left(a \wedge b, d_{2}\right) \\
& =f\left(a \wedge b, d_{3}\right) \wedge f\left(a \wedge b, d_{2}\right)=f\left(a \wedge b, d_{3} \wedge d_{2}\right) .
\end{aligned}
$$

But $a \wedge b=c \wedge b$, so

$$
\left[a, d_{1}\right] \wedge\left[b, d_{2}\right]=\left[c, d_{3}\right] \wedge\left[b, d_{2}\right] .
$$

Therefore $\Lambda$ is well defined in $(A \times D) / \theta$, and is clearly idempotent, commutative, and associative. Thus the system $((A \times D) / \theta, \leqq, \Lambda)$ is a semi-lattice.

We now show that $*$ is well defined on $(A \times D) / \theta$, and that it acts as an implication. Again let $a, b, c_{1}$, and $c_{2}$ be elements of $A$, and let $d_{1}, d_{2}, d_{3}$, and $d_{4}$ be elements of $D$ such that $\left[a, d_{1}\right]=\left[c_{1}, d_{3}\right]$, and $\left[b, d_{2}\right]=\left[c_{2}, d_{4}\right]$. Then $a=c_{1}, f\left(a, d_{1}\right)=f\left(a, d_{3}\right), b=c_{2}$, and $f\left(b, d_{2}\right)=f\left(b, d_{4}\right)$. So

$$
\begin{aligned}
{\left[a, d_{1}\right] *\left[b, d_{2}\right] } & =\left[a^{\prime} \vee b, f\left(a, d_{1} * d_{2}\right)\right] \\
& =\left[a^{\prime} \vee b, f\left(a, d_{1}\right) * f\left(a, d_{2}\right)\right] \\
& =\left[a^{\prime} \vee b, f\left(a, d_{3}\right) * f\left(a, d_{2}\right)\right] \\
& =\left[a^{\prime} \vee b, f\left(a, d_{3} * d_{2}\right)\right] .
\end{aligned}
$$

But

$$
f\left(b, d_{3} * d_{2}\right)=f\left(b, d_{3} * d_{4}\right),
$$

and since 


$$
a \wedge\left(a^{\prime} \vee b\right) \leqq b,
$$

it follows from (i) that

$$
f\left(a^{\prime} \vee b, f\left(a, d_{3} * d_{2}\right)\right)=f\left(a^{\prime} \vee b, f\left(a, d_{3} * d_{4}\right)\right)
$$

Hence

$$
\begin{aligned}
{\left[a, d_{1}\right] *\left[b, d_{2}\right] } & =\left[a^{\prime} \vee b, f\left(a, d_{3} * d_{4}\right)\right] \\
& =\left[c_{1}^{\prime} \vee c_{2}, f\left(c_{1}, d_{3} * d_{4}\right)\right]=\left[c_{1}, d_{3}\right] *\left[c_{2}, d_{4}\right]
\end{aligned}
$$

Hence * is well defined.

To show that $*$ is an implication, we need to show that

$$
\left[c, d_{3}\right] \leqq\left[a^{\prime} \vee b, f\left(a, d_{1} * d_{2}\right)\right]
$$

if and only if

$$
\left[c, d_{3}\right] \wedge\left[a, d_{1}\right] \leqq\left[b, d_{2}\right],
$$

for any $a, b$, and $c$ elements of $A$, and $d_{1}, d_{2}$, and $d_{3}$ elements of $D$. Assume that

$$
\left[c, d_{3}\right] \leqq\left[a^{\prime} \vee b, f\left(a, d_{1} * d_{2}\right)\right]
$$

Then by (ii), $c \leqq a^{\prime} \vee b$, and

$$
f\left(c, d_{3}\right) \leqq f\left(c, f\left(a, d_{1} * d_{2}\right)\right) .
$$

Hence $a \wedge c \leqq b$, and by (i),

$$
f\left(a \wedge c, d_{3}\right) \leqq f\left(a \wedge c, f\left(a, d_{1} * d_{2}\right)\right)=f\left(a \wedge c, d_{1} * d_{2}\right) .
$$

So since $f_{a \wedge c}$ is an endomorphism on $D$,

$$
f\left(a \wedge c, d_{1} \wedge d_{3}\right) \leqq f\left(a \wedge c, d_{2}\right),
$$

and so by (ii),

$$
\left[c, d_{3}\right] \wedge\left[a, d_{1}\right] \leqq\left[b, d_{2}\right] .
$$

Now assume that

$$
\left[c, d_{3}\right] \wedge\left[a, d_{1}\right] \leqq\left[b, d_{2}\right] .
$$

Then by (ii), $c \wedge a \leqq b$, and

$$
f\left(c \wedge a, d_{3} \wedge d_{1}\right) \leqq f\left(c \wedge a, d_{2}\right) .
$$

So $c \leqq a^{\prime} \vee b$, and since $f_{c \wedge a}$ is an endomorphism on $D$,

$$
f\left(c, d_{3}\right) \leqq f\left(c \wedge a, d_{3}\right) \leqq f\left(c \wedge a, d_{1} * d_{2}\right)=f\left(c, f\left(a, d_{1} * d_{2}\right)\right) .
$$

So

$$
\left[c, d_{3}\right] \leqq\left[a, d_{1}\right] *\left[b, d_{2}\right]
$$


So far we have shown that $(A \times D) / \theta$ is an implicative semi-lattice. Clearly $[0,1]$ is the lower bound of this system. Also for $[a, d] \in(A \times D) / \theta$,

$$
\begin{aligned}
{[a, d]^{*} } & =[a, d] *[0,1]=\left[a^{\prime}, f(a, d * 1)\right] \\
& =\left[a^{\prime}, f(a, 1)\right]=\left[a^{\prime}, 1\right] .
\end{aligned}
$$

Hence $[a, d]$ is dense if and only if $a=1$, and $[a, d]$ is closed if and only if $[a, d]=[a, 1]$. Also

$$
\left[1, d_{1}\right] *\left[1, d_{2}\right]=\left[1, f\left(1, d_{1} * d_{2}\right)\right]=\left[1, d_{1} * d_{2}\right]
$$

Therefore the mapping from $A$ into $(A \times D) / \theta$ which assigns to each $a \in A$ the element $[a, 1]$ in $(A \times D) / \theta$, and the mapping from $D$ into $(A \times D) / \theta$ which assigns to each $d \in D$, the element $[1, d]$ in $(A \times D) / \theta$ are both isomorphisms, the first onto the closed elements of $(A \times D) / \theta$, and the second onto the dense elements. Finally note that

$$
[a, 1] *[1, d]=\left[a^{\prime} \vee 1, f(a, 1 * d)\right]=[1, f(a, d)],
$$

for any $a \in A$ and $d \in D$.

Now let $L$ be a bounded implicative semi-lattice, with dense filter $D$. That $f(a, d)=a * d$ defines an admissible function from $L^{* *} \times D$ into $D$ follows from Lemma 2.1(i), (ii), (iv), (v), (vi) and (vii). Let $g$ be the function from $\left(L^{* *} \times D\right) / \theta$ into $L$ defined by $g([a, d])=a \wedge d$ for any $a \in L^{* *}$ and $d \in D$. We shall show that $g$ is well defined, and is an isomorphism onto $L$. Suppose that $[a, d]$ $=[b, e]$ for $a$ and $b$ elements of $L^{* *}$, and $d$ and $e$ elements of $D$. Then $a=b$ and $a * d=a * e$. Let $u=a \wedge d$ and $v=a \wedge e$. Then $u^{* *}=a=v^{* *}$, and so

Hence

$$
a * u=a *(a \wedge d)=a * d=a * e=a *(a \wedge e)=a * v
$$

$$
u=a \wedge(a * u)=a \wedge(a * v)=v,
$$

and therefore $g$ is well defined. That $g$ is onto follows from Theorem 4.4. Also for any $a$ and $b$ elements of $L^{* *}$, and $d$ and $e$ elements of $D$,

But

$$
\begin{aligned}
g([a, d] *[b, e]) & =g\left(\left[a^{*} \vee b, a *(d * e)\right]\right) \\
& =\left(a^{*} \vee b\right) \wedge(a *(d * e)) .
\end{aligned}
$$

$$
\begin{aligned}
g([a, d]) * g([b, e]) & =(a \wedge d) *(b \wedge e) \\
& =((a \wedge d) * b) \wedge((a \wedge d) * e) .
\end{aligned}
$$

But by Lemma 4.2(vi), since $b$ is an element of $L^{* *},(a \wedge d) * b=a^{*} \vee b$, and so $g([a, d]) * g([b, e])=g([a, d] *[b, e])$. To complete the proof, we observe that if $g([a, d])=1$ for some $a \in L^{* *}$ and $d \in D$, then $a=1$ and $d=1$. So $g$ is an isomorphism by Lemma 3.1 (iv). 
The following example shows that it is not possible to replace the word "semilattice" with "lattice" in the previous theorem. Let $A$ be the power set of a set of two elements $\{a, b\}$. Let $D$ be the set of real numbers $x$, such that $0<x \leqq 1$. Let $f(1, d)=d$ for $d \in D$, and $f(c, d)=1$ for $c \in A, c \neq 1$ and $d \in D$. Then $f$ is admissible, and $(A \times D) / \theta$ is a bounded implicative semi-lattice. Yet $(A \times D) / \theta$ is not a lattice, since $[a, 1]$ and $[b, 1]$ have no least upper bound in $(A \times D) / \theta$. This semi-lattice may be visualized by identifying the 1 of $A$ with the 1 of $D$, and then taking the ordinal sum of the remainder of $A$ with $D$.

6. Filters of bounded implicative semi-lattices. If $J$ is a filter of a bounded implicative semi-lattice $L$, then $J^{* *}$ is a filter of $L^{* *}$,

$$
J^{* *}=J \cap L^{* *},
$$

and

$$
J^{* *} \subset J \subset(* *)^{-1}\left(J^{* *}\right)
$$

If $K$ is a filter of $L^{* *}$, then $(* *)^{-1} K$ is a filter of $L$. Since the only solution of the equation $x^{* *}=0$ is $0, J$ is a proper filter of $L$ if and only if $J^{* *}$ is a proper filter of $L^{* *}$. Furthermore, if $J$ is an ultrafilter of $L$, then $J^{* *}$ is an ultrafilter of $L^{* *}$, and if $K$ is an ultrafilter of $L^{* *}$, then $(* *)^{-1} K$ is an ultrafilter of $L$. Also by a straightforward application of Zorn's lemma, it can be shown that every proper filter of a bounded implicative semi-lattice is contained in an ultrafilter.

Definition 6.1. A filter $J$ of a bounded implicative semi-lattice is total if and only if

$$
J=(* *)^{-1}\left(J^{* *}\right)
$$

Every filter $J$ is contained in a total filter $\bar{J}$ such that $J^{* *}=\bar{J}^{* *}$, and a filter is total if and only if $J=\bar{J}$. Every ultrafilter is total, and the mapping (**) induces a one-to-one correspondence between filters of $L^{* *}$ and total filters of $L$, which associates the ultrafilters of $L^{* *}$ with those of $L$. If $J$ is a filter of a bounded implicative semi-lattice $L$, and if $U$ is an ultrafilter of $L$ of which $J$ is a subset, then $J$ is a subset of $U$. From this and the fact that every filter of a Boolean algebra is an intersection of ultrafilters, it follows that a filter of $L$ is total if and only if it is an intersection of ultrafilters. From the fact that every Boolean algebra is semi-simple, it follows that the intersection of all of the ultrafilters of a bounded implicative semi-lattice is the filter of dense elements. Hence a bounded implicative semi-lattice is semi-simple if and only if it is a Boolean algebra.

THEOREM 6.2. Let L be a bounded implicative semi-lattice with dense filter $D$. Let $J$ be any filter of $L$. Then $J$ is total if and only if $D \subset J$. Hence $\bar{J}$ is the smallest filter containing $J \cup D$.

Proof. Clearly if $J$ is total, then $D \subset J$. Assume that $D \subset J$, and note that 
$J \subset \bar{J}$. Let $x \in \bar{J}$. Then $x^{* *} \in J^{* *}$, so there is some element $y \in J$ such that $x^{* *}=y^{* *}$. It follows from Corollary 4.5 that there is a dense element $d$ such that

$$
x \wedge d=y \wedge d .
$$

But $y \wedge d \in J$, so $x \wedge d \in J$, and hence $x \in J$. Thus $J=\bar{J}$.

For $x$ an element of an implicative semi-lattice $L$, let $x * L$ denote the set of elements $y$ of $L$ for each of which there is some element $z$ of $L$ such that

$$
y=x * z .
$$

LemMA 6.3. Let $\alpha$ be a homomorphism from an implicative semi-lattice $L$ onto a bounded implicative semi-lattice $L^{\prime}$. Let $J$ be the kernel of $\alpha$. Then for any $x \in L, \alpha(x)=0$ if and only if $x * L \subset J$.

Proof. Assume that $\alpha(x)=0$, and let $y \in x * L$. Then there is some $z \in L$ such that $y=x * z$. Hence

$$
\alpha(y)=\alpha(x) * \alpha(z)=0 * \alpha(z)=1 .
$$

So $y \in J$, and thus $x * L \subset J$. Now assume that $x * L \subset J$. Then for any $y \in L$, $\alpha(x) * \alpha(y)=1$. Hence $\alpha(x) \leqq \alpha(y)$, and so since $\alpha$ is onto $L^{\prime}, \alpha(x)=0$.

THEOREM 6.4. Let $J$ be a filter of a bounded implicative semi-lattice $L$. Then $J$ is an ultrafilter if and only if $L / J$ is simple.

Proof. First assume that $J$ is an ultrafilter, and that $x \in L-J$. Then since $J$ is total, $x^{* *} \notin J^{* *}$, so since $J^{* *}$ is an ultrafilter of the Boolean algebra $L^{* *}, x^{*} \in J^{* *} \subset J$. Thus since $x \wedge x^{*}=0, x / J=0 / J$, and hence $L / J$ is simple. Now assume that $L / J$ is simple, and let $U$ be a filter of $L$, properly containing $J$. Let $x \in U-J$. Then, letting $\pi$ denote the homomorphism which assigns to each $y \in L$ the element $y / J$ of $L / J, \pi(x)=0$, so by Lemma $6.3, x * 0=x^{*} \in J$. Hence

$$
0=x \wedge x^{*} \in U,
$$

and so $U$ is not proper. Thus $J$ is an ultrafilter.

Corollary 6.5. Let $L$ be a bounded implicative semi-lattice with dense filter $D$. Then $D$ is an ultrafilter if and only if $L^{* *}$ is simple.

Let $\alpha$ be a homomorphism from a bounded implicative semi-lattice $L$, with dense filter $D$ onto a bounded implicative semi-lattice $L^{\prime}$ with dense filter $D^{\prime}$. Let $J$ be the kernel of $\alpha$. Then $\alpha \mid L^{* *}$ is a Boolean homomorphism with kernel $L^{* *} \cap J$, and $\alpha \mid D$ is a homomorphism with kernel $D \cap J$. For any subsets $X$ and $Y$ of $L$, let $X \wedge Y$ denote the set of elements $z$ of $L$ for each of which there is an element $x$ of $X$, and an element $y$ of $Y$ such that

Then clearly

$$
z=x \wedge y \text {. }
$$




$$
J=(L * * \cap J) \wedge(D \cap J) .
$$

By Theorem 5.2, there is an admissible function associated with each bounded implicative semi-lattice $L$. For $a \in L^{* *}$, we shall refer to the $a$-classes of this function as $a$-classes of $L$.

THEOREM 6.6. Let L be a bounded implicative semi-lattice with dense filter $D$. Let $\alpha$ be a Boolean homomorphism with domain $L^{* *}$. Let $\beta$ be a homomorphism with domain $D$. Then a necessary and sufficient condition that there be a homomorphism with domain $L$, the reduction of which to $L^{* *}$ is $\alpha$ and the reduction of which to $D$ is $\beta$, is that for every element a in the kernel of $\alpha$, the kernel of $\beta$ be a union of a-classes of L. Furthermore, if this extension exists, it is unique.

REMARK. Note that this theorem does not assert that suitable homomorphisms from the closed algebra and dense filter of a semi-lattice $L$ onto those of a semilattice $L^{\prime}$ can be extended from $L$ to $L^{\prime}$, but rather that there is a semi-lattice $L^{\prime \prime}$ to which $L$ is homomorphic, and having the image algebra and image semilattice as closed algebra and dense filter.

Proof. Necessity is obvioús. To show sufficiency, it suffices to show the following: If $B$ is a filter of $L^{* *}$, and $E$ is a filter of $D$ such that for every $a \in B$, and every $d_{1} \in E$ and $d_{2} \in D$, if

$$
a * d_{1}=a * d_{2},
$$

then $d_{2} \in E$, and if

then

$$
J=B \wedge E,
$$

(i) $A \cap J=B$,

(ii) $D \cap J=E$,

(iii) $J$ is a filter of $L$.

(i) Since $1 \in E, B \subset A \cap J$. Let $x \in A \cap J$. Then $x=a \wedge e$ for some $a \in B$, and $e \in E$. Also

$$
x=x^{* *}=a^{* *} \wedge e^{* *}=a^{* *}=a,
$$

so $x \in B$. Thus $B=A \cap J$.

(ii) Again, since $1 \in B, E \subset D \cap J$. For $x \in D \cap J$, there are elements $a \in B$ and $e \in E$ such that $x=a \wedge e$. But $x^{* *}=1$, so

$$
1=a^{* *} \wedge e^{* *}=a,
$$

and thus $x=e \in E$. Hence $E=D \cap J$.

(iii) Clearly if $x$ and $y$ are elements of $J$, then $x \wedge y \in J$. Suppose that $x \in J$, and $y \in L$ such that $y \geqq x$. Then $x=a \wedge e$ for some $a \in B$ and $e \in E$. Hence 


$$
x^{* *}=a^{* *} \wedge e^{* *}=a,
$$

and so $x^{* *} \in B$. But since $y^{* *} \geqq x^{* *}, y^{* *} \in B$. Now

$$
y=y^{* *} \wedge\left(y^{* *} * y\right) \text {. }
$$

Also, in the representation of $x$ given above, $e \leqq x^{* *} * x$ by Theorem 4.4. So $x^{* * *} x \in E$. But since $y \geqq x$,

$$
x^{* *} * x \leqq x^{* *} * y
$$

and so $x^{* *} * y \in E$. Now

$$
x^{* *} * y=\left(x^{* *} \wedge y^{* *}\right) * y=x^{* *} *\left(y^{* *} * y\right),
$$

so

$$
x^{* * *} *(x * * * y)=x^{* * *} *\left(y^{* * *} * y\right),
$$

and so $y^{* *} * y \in E$. Thus $y \in J$ and $J$ is a filter.

COROLlary 5.7. Let $\alpha$ be a homomorphism, the domain of which is a bounded implicative semi-lattice $L$, with dense filter $D$. Then $\alpha$ is an isomorphism if and only if both $\alpha \mid L^{* *}$ and $\alpha \mid D$ are isomorphisms.

\section{REFERENCES}

1. Garrett Birkhoff, Lattice theory, Amer. Math. Soc. Colloq. Publ. Vol. 25, rev. ed., Amer. Math. Soc., Providence, R. I., 1948.

2. H. B. Curry, Foundations of mathematical logic, McGraw-Hill, New York, 1963.

3. J. C. C. McKinsey and A. Tarski, On closed elements in closure algebras, Ann. of Math. (2) 47 (1946), 122-162.

4. Orrin Frink, Pseudo-complements in semi-lattices, Duke Math. J. 29 (1962), 505-514.

5. V. Glivenko, Sur quelques points de la logique de M. Brouwer, Bull. Acad. Sci. Belg. 15 (1929), 183-188.

6. António Monteiro, Axiomes indépendants pour les algèbres de Brouwer, Rev. Un. Mat. Argentina 17 (1955), 149-160.

7. M. H. Stone, Topological representations of distributive lattices and Brouwerian logics, Cas. Mat. Fys. 67 (1937), 1-25.

SOUTHWESTERN AT MEMPHIS,

MEMPHIS, TENNESSEE

. $\ln \mathrm{i}=\mathrm{d}$ eurl. $\mathrm{a} \ni \%$ ve

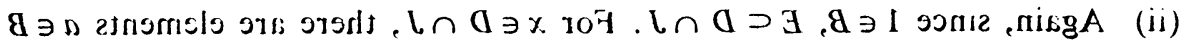

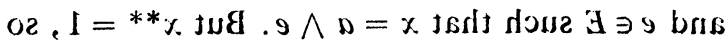

$$
\begin{aligned}
& D=* *^{2} \wedge *_{D}=1
\end{aligned}
$$

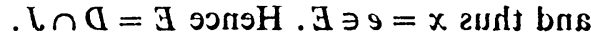

. ᄂ

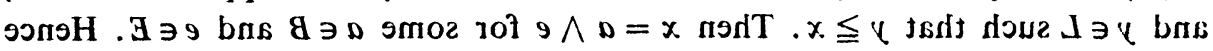

